\title{
Sum Rules for Parity Violating Compton Amplitudes.
}

\author{
Leszek Łukaszuk \\ Andrzej Soltan Institute for Nuclear Studies, Hoza 69, 00-681 Warsaw,Poland
}

\begin{abstract}
Sum rules for parity violating spin polarizabilities are derived and discussed. They hold both for hadron and nuclear stable targets of arbitrary spin and are exact in strong interactions. Examples of applications to the cases of proton and deuteron targets are given. The legitimacy of the dispersive approach in the Standard Model is discussed.
\end{abstract}

Key words: Forward Compton amplitudes; Parity violation; Asymptotic states in Standard Model; Sum rules; Superconvergence hypothesis; Spin polarizabilities

PACS: 12.15.Ji, 13.60.Fz, 24.80.+y, 25.20.Dc, 25.20.Lj 


\section{Introduction}

The recent revival [1-5] of interest in the weak part of photon - hadron interactions seems to be closely connected with the advent of intense polarized beams of photons [6-10]. Generally it can be expected that future experiments will be a good source of information in the theoretically difficult domain of low energy hadronic structure. For example the threshold production asymmetries in pion photoproduction on proton [1,2] would test the consistency of the weak $N N \pi$ coupling,$h_{\pi N N}^{(1)}$ with the "best fit" coupling scheme [11]. Similiar expectations are connected with low energy Compton scattering $[3,4]$ on a proton. The $h_{\pi N N}^{(1)}$ problem is acute because results from different nuclear $[12,13]$ and atomic [14] physics experiments seem to indicate quite different values of $h_{\pi N N}^{(1)}$. The theoretical interpretation of future experiments on more elementary targets should be easier, of course. At the moment neither the large nor the small $h_{\pi N N}^{(1)}$ option can be excluded and it is possible that this constant could be much smaller $[2,5]$ then the so called "best value" [11] ; then the short distance (in comparison with effective $\pi$ exchange) Parity Violating (p.v.) contributions should be larger than those from the set of "best values" [11] - here a test of the importance of such contributions would be the photodisintegration of deuterons [5]. Both real and virual photon -initiated effects are of interest and have been considered in the literature $[1-5,15,16]$. Experimentally these cases correspond to photon and electron -initiated collisions, respectively. A convenient feature of (real) photon initiated collisions is the absence of direct $Z$ exchange between projectile and target so it is a unique situation where the p.v. structure of the electromagnetic current itself is singled out without further elaborations. On the other hand disentangling virtual photon p.v. contributions from electroproduction seems to be difficult - already at $Q^{2}>0.1 G e V^{2}$ it's contribution to the measured asymetry is a few percent of the neutral current's contribution [15]. This situation reflects the fact that p.v. interference terms involving only electromagnetic currents must contain an additional photon propagator so an extra $(\alpha)$ factor appears when compared with terms which contain electromagnetic and neutral current exchanges (Only for $Q^{2} \rightarrow 0$ will the additional photon propagator dominate for $\pi$ meson electroproduction it takes place below $Q^{2}$ of the order of $10^{-3} \mathrm{GeV}^{2}$ [15]). Having this situation in mind we shall limit ourselves to real photons only in the further discussion so that in the language of ref. [15] we shall confine ourselves to the p.v. electromagnetic and nuclear interaction at $Q^{2}=0$. Model dependent estimates of these interactions lead to asymmetries of the order of $10^{-7}$ for low energy photoproduction [1,15] ("best fit " coupling scheme). The Compton amplitude asymmetries have been theoretically estimated in the case of proton target as $10^{-8}$ within the one-loop approximation of heavy-baryon chiral perturbation theory $(\mathrm{HB} \chi \mathrm{PT})[3,4]$. As it was shown $[17,18]$ that p.v. effects at low energies, $\omega$, do not contribute to the static 
terms (i.e. they are at least of order $O\left(\omega^{2}\right)$ ) in this process, we can expect that these p.v. asymmetries and p.v. spin polarizabilities should be closely related. Therefore it is sensible to question the credibility of $\mathrm{HB} \chi \mathrm{PT}$ in the context of parity conserving (p.c.) spin polarizabilities of the nucleon [26]; in fact the $\mathrm{HB} \chi \mathrm{PT}$ expansion was shown to be an unstable method for the calculation of these polarizabilities [19-22]. So in the p.v. case, apart from the uncertainty of couplings, this kind of unstability cannot be ruled out. It would be therefore appropriate to apply the dispersive approach, successful in p.c. analysis $[23,6]$, using a low energy multipole expansion and in such a way limiting the number of adjustable parameters - however, in p.c. analysis it was shown that apart from general theoretical input, quite acurate experimental data are needed to determine these parameters [23]. We have no comparable data for the p.v. analysis at the moment, so it seems sensible to try to correlate different p.v. hadronic or nuclear observables via model independent relations such as dispersive sum rules, in analogy with those applied to the spin dependent p.c. domain [24-27]. We shall limit ourselves to the sum rules for forward amplitudes, as in this case relations between physically measured cross sections are most transparent. In what follows we shall discuss the legitimacy of the relevant dispersion relations, apply them to Compton scattering on protons and deuterons using existing models for p.v. in photoproduction $[1,15]$ and photo-disintegration [5] . We shall also discuss the problem of subtractions in the context of the eventual use of superconvergence assumptions. Tentative predictions made under such assumptions will be formulated.

\section{Asymptotic States in Standard Model}

As we want to discuss dispersion formulae for collision amplitudes, it is a suitable place to ask to what degree the usual properties of these amplitudes (existence of asymptotic states and of interpolating local fields) are exhibited in the Standard Model. The asymptotic states have to correspond to a Fock space of stable particles, so we are left with photons, electrons, neutrinos (at least the lightest one ), protons and stable atomic ions. Let us mention here that the existence of unstable fields is a source of concern in Quantum Field Theory $[28,29]$. Next, each stable particle should correspond to an irreducible Poincare (unitary) representation and here trouble appears with charged particles [30,31]. This is connected with QED infrared radiation and a well defined procedure exists in perturbative calculus only. This is the reason why our considerations concerning Compton amplitudes will be limited to the order $\alpha$ in the p.c. part and to order $\alpha^{2}$ in the p.v. part (they are infrared safe and at low energies are $\alpha G_{F}$ order contributions). Still we are left with the problem of asymptotic states and interpolating fields in the QCD part of the SM - we shall

rely on the results of Oehme: "the analytic properties of physical amplitudes 
are the same as those obtained on the basis of an effective theory involving only the composite, physical fields" [32] (in other words confinement does not spoil the old axiomatic proofs for hadronic interactions [33] ).

\section{Parity Violating Compton Sum Rules for Arbitrary Target}

\subsection{Dispersion Relations and Low Energy Behaviour}

Working in the lowest electroweak order it is reasonable to abandon $\mathrm{C}, \mathrm{P}$ invariance only and keep in our considerations T-invariance. The strong part of the interactions is taken without any approximation. The analyticity of the forward Compton amplitude and crossing properties follow from the typical steps sketched below. We start from LSZ - derived formula [34] for the forward Compton amplitude; for convenience we choose the target in the lab frame and drop inessential for the further argument contact terms, so that we get [34]

$$
\begin{gathered}
S_{f i}=I+i(2 \pi)^{4} \delta_{4}\left(P_{f}-P_{i}\right) M_{f i} \\
M_{f i}=e^{2} \overline{\epsilon_{f}^{\mu}} \epsilon_{i}^{\nu} T_{\mu \nu} \\
T_{\mu \nu}\left(q, M, s_{f}, s_{i}\right)=i \int d^{4} x e^{i \omega\left(x_{0}-\vec{n} \vec{x}\right)} \Theta\left(x_{0}\right)\left\langle M, s_{f}\left|\left[j_{\mu}(x), j_{\nu}(0)\right]\right| M, s_{i}\right\rangle
\end{gathered}
$$

where $\vec{q}=\omega \vec{n}$ is the photon's momentum and with z-axis taken in it's direction, $\vec{n}$, $s_{i}, s_{f}$ is the target's initial (final) z-component of spin, $\epsilon_{i}, \epsilon_{f}$ denote initial (final) circular polarization states of the photon. Let us work in radiation gauge so only the $\mu, \nu=1,2$ components of $j_{\mu}, j_{\nu}$ contribute. In what follows we never use parity conservation.

The analyticity in the upper complex half-plane of $\omega$ follows from the fact that the retarded commutator in (3.3) vanishes for $x_{0}<0$ and, due to causality, vanishes also for $x_{0}<|z|$. For Rew $>0$ approaching the real axis we get the physical Compton amplitude specified by (3.2). For Rew $<0$ the limiting amplitude can be obtained by applying complex conjugation to (3.3) and exploiting invariance of the matrix elements with respect to rotations; here rotation around the $\mathrm{y}$ or $\mathrm{x}$-axis by angle $\pi$ should be used. The result, independent of $\mathrm{P}, \mathrm{C}, \mathrm{T}$ invariances, reads

$$
M_{h_{f}, h_{i}}^{s_{f}, s_{i}}(-R e \omega+i \epsilon)=\overline{M_{-h_{f},-h_{i}}^{s_{i}, s_{f}}(R e \omega+i \epsilon)}
$$

Demanding $\mathrm{T}$ invariance we get

$$
M_{h_{f}, h_{i}}^{s_{f}, s_{i}}(-R e \omega+i \epsilon)=\overline{M_{-h_{i},-h_{f}}^{s_{f}, s_{i}}(R e \omega+i \epsilon)}
$$


In what follows we shall be interested in coherent amplitudes only (i.e. $s_{i}=s_{f}$ and $h_{i}=h_{f}$ ), suitable for sum rules, as their imaginary parts are proportional to the total cross sections. In this case (3.4) and (3.5) are eqiuvalent, so the demand of $\mathrm{T}$ invariance is not necessary, however, this invariance will be used below in the estimates of the low energy behaviour. We shall use abbreviated names $f$ for these amplitudes

$$
f_{s, h}(\omega)=M_{h, h}^{s, s}(\omega)
$$

so that

$$
f_{s, h}(-R e \omega+i \epsilon)=\overline{f_{s,-h}(\operatorname{Re} \omega+i \epsilon)}
$$

We shall use amplitudes [35] normalized such that, for any target

$$
\operatorname{Im} f_{s, h}(\omega)=\omega \sigma_{s, h}^{T}(\omega)
$$

Then analyticity, crossing (3.7) and unitarity lead, through Hilbert formulae to the dispersion relations for these amplitudes

$$
R e f_{s, h}(\omega)=\frac{1}{\pi} P \int_{\omega_{t h}}^{\infty} \frac{\omega^{\prime} \sigma_{s, h}^{T}\left(\omega^{\prime}\right)}{\omega^{\prime}-\omega} d \omega^{\prime}+\frac{1}{\pi} \int_{\omega_{t h}}^{\infty} \frac{\omega^{\prime} \sigma_{s,-h}^{T}\left(\omega^{\prime}\right)}{\omega^{\prime}+\omega} d \omega^{\prime}+(s u b t r .)
$$

On the other hand any amplitude $f$ can be written as

$$
f_{s, h}=f_{s, h}^{+}+f_{s, h}^{-}
$$

where $f^{+}, f^{-}$are p.c. and p.v., respectively,

$$
f_{s, h}^{ \pm}=\frac{1}{2}\left(f_{s, h} \pm f_{-s,-h}\right)
$$

There exist proofs of low energy QED theorems for targets of any spin $[35,36]$ up to $O(\omega)$ terms. Explicit proof that the p.v. amplitudes of SM are of order $O\left(\omega^{2}\right)$ has been given for spin $\frac{1}{2}[17,18]$. I learned from I.B.Khriplovich that this result holds for targets of any spin if one neglects, as we do, T-violation; the reason is that the leading (larger than $O\left(\omega^{2}\right)$ ) low energy behaviour comes from the pole diagrams and that in this case the only p.v. coupling of a real photon involves electric dipole moment [37]. Therefore we can write, for a target of any spin

$$
M_{h_{f}, h_{i}}^{s_{f}, s_{i}}(\omega \rightarrow 0)=\delta_{h_{f}, h_{i}} \delta_{s_{f}, s_{i}} f_{s_{i}, h_{i}}^{(+) L E T}+O\left(\omega^{2}\right)=M^{(+)}+O\left(\omega^{2}\right)
$$


with $f^{(+) L E T}$ known from the p.c. Low Energy Theorems [35,36] and

$$
M_{h_{f}, h_{i}}^{( \pm) s_{f}, s_{i}}=\frac{1}{2}\left(M_{h_{f}, h_{i}}^{s_{f}, s_{i}} \pm M_{-h_{f},-h_{i}}^{-s_{f},-s_{i}}\right)
$$

so that

$$
\left.f_{s, h}^{-}(\omega)\right|_{\omega \rightarrow 0}=O\left(\omega^{2}\right)
$$

Hence for any target in the limit $\omega \rightarrow 0$ the ratio

$$
A\left(s_{i}, h_{i}\right)=\frac{\sum_{s_{f}, h_{f}}\left(\left|M_{h_{f}, h_{i}}^{s_{f}, s_{i}}\right|^{2}-\left|M_{h_{f},-h_{i}}^{s_{f},-s_{i}}\right|^{2}\right)}{4 f_{s_{i}, h_{i}}^{(+) L E T}}=f_{s_{i}, h_{i}}^{-}+O\left(\omega^{4}\right)
$$

measures the parity violating part of the forward amplitude. This came out to be simple for any target due to the diagonal form of $M^{+}$at low energies (comp. (3.12)).

It will be convenient to consider p.v. amplitudes averaged over the spin of the target

$$
f_{h}^{(-) \gamma}=\frac{1}{2 S+1} \sum_{s_{i}} f_{s_{i}, h}^{-}
$$

and averaged over the photon's helicity

$$
f_{s}^{(-) t g}=\frac{1}{2}\left(f_{s,+1}^{-}+f_{s,-1}^{-}\right)
$$

These amplitudes are expressed by integrals over relevant differences of the total cross sections (comp. (3.9)).

$$
R e f_{h}^{(-) \gamma}=\frac{\omega}{\pi} P \int_{\omega_{t h}}^{\infty} \frac{\omega^{\prime}}{\omega^{\prime 2}-\omega^{2}}\left(\sigma_{h}^{T}-\sigma_{-h}^{T}\right) d \omega^{\prime}+(\text { subtr. })
$$

where

$$
\sigma_{h}^{T}=\frac{1}{2 S+1} \sum_{s_{i}} \sigma_{s_{i}, h}^{T}
$$

and

$$
R e f_{s}^{(-) t g}=\frac{1}{\pi} P \int_{\omega_{t h}}^{\infty} \frac{\omega^{\prime 2}}{\omega^{\prime 2}-\omega^{2}}\left(\sigma_{s}^{T}-\sigma_{-s}^{T}\right) d \omega^{\prime}+(\text { subtr. })
$$


where

$$
\sigma_{s}^{T}=\frac{1}{2}\left(\sigma_{s,+1}^{T}+\sigma_{s,-1}^{T}\right)
$$

\subsection{Sum Rules for p.v. spin polarizabilities}

As the p.v. amplitudes considered in our approximation are infrared safe we are entitled to expect that the high energy growth of forward amplitudes will be at most $\omega(\ln \omega)^{2}$ as results from general principles for finite range interactions [33]. Condition (3.12) means that for a target of any spin no

arbitrary constants appear in the dispersion formulae for $f^{(-) t g}, f^{(-) \gamma}$ if the subtraction point is taken at $\omega=0$, therefore

$$
R e f_{s}^{(-) t g}=\frac{\omega^{2}}{\pi} P \int_{\omega_{t h}}^{\infty} \frac{\sigma_{s}^{T}-\sigma_{-s}^{T}}{\omega^{\prime 2}-\omega^{2}} d \omega^{\prime}=-4 \pi \omega^{2} a_{s}^{(-) t g}(\omega)
$$

and

$$
R e f_{h}^{(-) \gamma}=\frac{\omega^{3}}{\pi} P \int_{\omega_{t h}}^{\infty} \frac{\sigma_{h}^{T}-\sigma_{-h}^{T}}{\omega^{\prime}\left(\omega^{\prime 2}-\omega^{2}\right)} d \omega^{\prime}=-4 \pi \omega^{3} a_{h}^{(-) \gamma}(\omega)
$$

For $\omega \rightarrow 0$ eqns.(3.22, 3.23) yield sum rules for p.v. forward polarizabilities $a_{s}^{(-) t g}(\omega=0), a_{h}^{(-) \gamma}(\omega=0)$ defined in analogy with p.c. forward spin polarizabilities [6]

$$
\begin{aligned}
& a_{s}^{(-) t g}(0)=\frac{1}{4 \pi^{2}} \int_{\omega_{t h}}^{\infty} \frac{\sigma_{-s}^{T}-\sigma_{s}^{T}}{\omega^{\prime 2}} d \omega^{\prime} \\
& a_{h}^{(-) \gamma}(0)=\frac{1}{4 \pi^{2}} \int_{\omega_{t h}}^{\infty} \frac{\sigma_{-h}^{T}-\sigma_{h}^{T}}{\omega^{\prime 3}} d \omega^{\prime}
\end{aligned}
$$

\subsection{Superconvergence Hypothesis}

If we assume superconvergence of the type $\frac{f(z)}{z} \rightarrow 0$ at infinity for the asymmetric amplitude (3.23), then the p.v. analogue of DHG [24,25] is obtained

$$
\int_{\omega_{t h}}^{\infty} \frac{\sigma_{h}^{T}-\sigma_{-h}^{T}}{\omega^{\prime}} d \omega^{\prime}=0
$$


It is natural to question the legitimacy and consequences of such an assumption for the p.v. amplitude $f^{(-) \gamma}$. Consequences of this formula will be mentioned in the context of model dependent applications in the next section. The check whether at least in the perturbative QCD regime the relevant contributions to the total cross sections asymptotically cancel for a given target in (3.26)has not yet been done. One should calculate a few different processes which might conspire to give vanishing overall difference of the total cross sections. It might also happen that the non perturbative regime plays an equal or essential role. The seemingly plausible conjecture that spin dependent contributions to the integrated cross sections asymptotically vanish for any reaction, need not be true. We have checked [38] that a class of $\gamma$-induced processes with polarized target (proton), namely those with the production of additional quark antiquark pair via unpolarized photon structure [39] yields

a non vanishing (in fact slowly rising)contribution to the difference $\sigma_{\frac{1}{2}}^{T}-\sigma_{-\frac{1}{2}}^{T}$ if present-day parton distributions are used [40]. Despite this warning we shall study the consequences of (3.26) in the next section.

\section{Examples of Applications}

\subsection{Proton Target}

The p.v. Compton amplitude can be written in the c.m.s. as $[3,4]$

$$
\begin{aligned}
& M_{h_{f}, h_{i}}^{(-) s_{f}, s_{i}}\left(\vec{k}, \overrightarrow{k^{\prime}}\right)=\overline{N_{s_{f}}}\left[F_{1} \vec{\sigma} \cdot\left(\hat{\vec{k}}+\hat{\overrightarrow{k^{\prime}}}\right) \overrightarrow{\epsilon_{i}} \cdot \overrightarrow{\overrightarrow{\epsilon_{f}^{\prime}}}-F_{2}\left(\vec{\sigma} \cdot \overrightarrow{\overrightarrow{\epsilon_{f}^{\prime}}} \hat{\overrightarrow{k^{\prime}}} \cdot \overrightarrow{\epsilon_{i}}+\vec{\sigma} \cdot \overrightarrow{\epsilon_{i}} \cdot \overrightarrow{\overrightarrow{k^{\prime}}} \cdot \overrightarrow{\overrightarrow{\epsilon_{f}^{\prime}}}\right)\right. \\
& \left.-F_{3} \hat{\vec{k}} \cdot \overrightarrow{\overrightarrow{\epsilon_{f}^{\prime}}} \hat{\overrightarrow{k^{\prime}}} \cdot \overrightarrow{\epsilon_{i}} \vec{\sigma} \cdot\left(\hat{\vec{k}}+\hat{\overrightarrow{k^{\prime}}}\right)-i F_{4} \overrightarrow{\epsilon_{i}} \times \overrightarrow{\overrightarrow{\epsilon_{f}^{\prime}}} \cdot\left(\hat{\vec{k}}+\hat{\overrightarrow{k^{\prime}}}\right)\right] N_{s_{i}}
\end{aligned}
$$

so that

$$
\begin{gathered}
f_{\frac{1}{2}}^{(-) p}=2 F_{1}=O\left(\omega^{2}\right) \\
f_{h=+1}^{(-) \gamma}=-2 F_{4}=O\left(\omega^{3}\right)
\end{gathered}
$$

The $\mathrm{HB} \chi \mathrm{PT}$ analysis $[3,4]$ provides values of coefficients $F_{1}, F_{4}$

$$
\left.F_{1}\right|_{\omega \rightarrow 0}=-\frac{e^{2}}{M}\left(\frac{\omega}{m_{\pi}}\right)^{2} \frac{M}{F_{\pi}} \frac{g_{A} h_{\pi N N}^{(1)}}{24 \sqrt{2} \pi^{2}}
$$




$$
\left.F_{4}\right|_{\omega \rightarrow 0}=\frac{e^{2}}{M}\left(\frac{\omega}{m_{\pi}}\right)^{3} \frac{m_{\pi}}{F_{\pi}} \frac{g_{A} h_{\pi N N}^{(1)} \mu_{n}}{24 \sqrt{2} \pi^{2}}
$$

where $F_{\pi}=93 \mathrm{MeV}, \quad g_{A}=1.26, \quad \mu_{n}=-1.91, \quad h_{\pi N N}^{(1)} \simeq 5 \cdot 10^{-7}$ in the "best fit" parametrization, $M$ is the nucleon mass. On the other hand there are theoretical results for p.v. effects in near threshold photoproduction $[1,2,15]$. These values could be used in our relations (3.22, 3.23). Denoting

$$
\begin{aligned}
R e f_{s=\frac{1}{2}}^{(-) p} & =-\left.\frac{e^{2}}{M} \beta^{-}\left(\frac{\omega}{m_{\pi}}\right)^{2}\right|_{\omega \rightarrow 0} \\
R e f_{h=+1}^{(-) \gamma} & =-\left.\frac{e^{2}}{M} \gamma^{-}\left(\frac{\omega}{m_{\pi}}\right)^{3}\right|_{\omega \rightarrow 0}
\end{aligned}
$$

we have

$$
\begin{aligned}
& a_{\frac{1}{2}}^{(-) t g}(0)=\frac{\alpha}{M m_{\pi}^{2}} \beta^{-}=3.2 \beta^{(-)} 10^{-3}[\mathrm{fm}]^{3} \\
& a_{1}^{(-) \gamma}(0)=\frac{\alpha}{M m_{\pi}^{3}} \gamma^{-}=4.5 \gamma^{(-)} 10^{-3}[\mathrm{fm}]^{4}
\end{aligned}
$$

with

$$
\begin{gathered}
\beta^{-}=-\left(\frac{e^{2}}{M m_{\pi}^{2}}\right)^{-1} \frac{1}{\pi} \int_{\omega_{t h}}^{\infty} \frac{\sigma_{s=\frac{1}{2}}^{T}-\sigma_{s=-\frac{1}{2}}^{T}}{\omega^{\prime 2}} d \omega^{\prime} \\
\gamma^{-}=-\left(\frac{e^{2}}{M m_{\pi}^{3}}\right)^{-1} \frac{1}{\pi} \int_{\omega_{t h}}^{\infty} \frac{\sigma_{h=+1}^{T}-\sigma_{h=-1}^{T}}{\omega^{\prime 3}} d \omega^{\prime}
\end{gathered}
$$

while $\beta_{\chi}, \gamma_{\chi}$ coming from $(4.4,4.5)$ are

$$
\begin{gathered}
\beta_{\chi}^{-}=\frac{M}{F_{\pi}} \frac{g_{A} h_{\pi N N}^{(1)}}{12 \sqrt{2} \pi^{2}} \simeq 4 \cdot 10^{-8} \\
\gamma_{\chi}^{-}=\mu_{n} \frac{m_{\pi}}{F_{\pi}} \frac{g_{A} h_{\pi N N}^{(1)}}{12 \sqrt{2} \pi^{2}} \simeq-1 \cdot 10^{-8}
\end{gathered}
$$

We shall use cross sections from $[1,15]$ in our sum rules, compare consistency and finally, assuming superconvergence (3.26) discuss posible consequences. Let us start with the $\mathrm{HB} \chi \mathrm{PT}$ approach to photoproduction; taking dominant at threshold terms in the effective lagrangian of reference [1] we integrate 
them up to $\omega=200 \mathrm{MeV}$ i. e. in the region where $\mathrm{HB} \chi \mathrm{PT}$ should be reliable. The results are $1 \cdot 10^{-8}$ for $\beta^{-}$and $-4 \cdot 10^{-9}$ for $\gamma^{-}$, to compare with $4 \cdot 10^{-8}$ and $-1 \cdot 10^{-8}$ for $\beta_{\chi}^{-}$and $\gamma_{\chi}^{-}$, respectively. This means that in $(\gamma p \longrightarrow \gamma p)$ $\mathrm{HB} \chi \mathrm{PT}$ calculations $[3,4]$ contributions from much higher energies have been involved. Indeed, extrapolating the threshold behaviour up to $1 \mathrm{GeV}$ and inserting into (4.10), (4.11) we get values which compare well with results from $[3,4]$. However, there is no reason to assume the validity of threshold type behaviour in such a large region. Therefore we turn to an analysis [15] where elaborated Born type exchanges (with resonances and form factors considered) were put together. We shall consider the "best fit" predictions contained in figs $11-15$ of [15]. Using eqs. (4.10), (4.11) we get $-5 \cdot 10^{-9}$ for $\beta^{-}$and $-1 \cdot 10^{-8}$ for $\gamma^{-}$. This is an example of the usefulness of measurements, not only of threshold p.v. photoproduction, but low energy Compton asymmetries, too, in future experiments, as they can shed some light on the existence of structure at higher energies. In the comparison made above the $\beta^{-}$obtained differ by an order of magnitude. This is a reflection of the quite different behaviour of cross sections. Let us pass now to the superconvergence hypothesis (3.26). The contributions in the region below $0.55 \mathrm{GeV}$ we calculate from [15] and obtain the relation

$$
\int_{0.55 \mathrm{GeV}}^{\infty} \frac{\sigma_{1}^{T}-\sigma_{-1}^{T}}{\omega^{\prime}} d \omega^{\prime} \simeq-30 p b
$$

If we further assume - by analogy with the gross features of DHG sum rule saturation - that the necessary contribution comes from the region below 1 $\mathrm{GeV}$ we get for the average asymmetry in the region $(0.55-1 \mathrm{GeV})$ a value $(-50 \mathrm{pb})$. This might indicate that it is desirable to look for p.v. effects in this region.

\subsection{Deuteron Target}

We shall consider the model of Khriplovich and Korkin [5] for p.v. effects in photodisintegration. As emphasized in ref. [5], this process may provide a test for the importance of short distance (in comparison with $\pi$ exchange) Parity Violating contributions. Here the difference of cross sections, integrated up to $10 \mathrm{MeV}$, yields a rather small contribution in natural units (i.e. $\frac{\omega}{2.23 \mathrm{MeV}}$ ) but compared with that for proton i.e. in units $\frac{\omega}{m_{\pi}}$ it is large:

$$
R e f_{h=+1}^{(-) \gamma}=-\left.\frac{e^{2}}{M_{D}} \gamma_{D}^{-}\left(\frac{\omega}{m_{\pi}}\right)^{3}\right|_{\omega \rightarrow 0}
$$


with

$$
\gamma_{D}^{-} \simeq-2 \cdot 10^{-4}
$$

If we use a dispersion relation close to the disintegration threshold $\omega \simeq$ $2.23 \mathrm{MeV}$, we get

$$
-\left.R e f_{+1}^{(-) \gamma}(\omega) \cdot\left(\frac{e^{2}}{M_{D}}\right)^{-1}\right|_{\omega \rightarrow 2.23 M e V} \simeq-1 \cdot 10^{-8}
$$

while extrapolation of $(4.15)$ would give $-7 \cdot 10^{-10}$. This indicates that p.v. effects are strengthened by an order of magnitude due to the cusp effect.

\section{Concluding remarks}

New sum rules derived in Section3 were found helpful in checking the consistency of various theoretical approaches (see discussion of the example in Section4.1). Moreover, under the superconvergence hypothesis (see Section.3.3) p.v. effects in photoproduction far from the threshold (see Section.4.1) can be estimated from the low energy data (models). The subject of superconvergence seems to be a challenge for further theoretical studies.

As our sum rules and dispersive formulae hold for stable nuclear targets of arbitrary spin, future applications to complex nuclei are of interest. In this context the cusp enhancement of p.v. effects on the deuteron (see Section4.2) indicates that Compton scattering at energies close to the nuclear inelastic thresholds should be of importance.

\section{Acknowledgements}

I would like to thank Professors: Mamoru Fujiwara, Elliot Leader and Ziemowid Sujkowski for encouragement and discussions on photon induced processes, Youlik Khriplovich for enlightment on LET in SM and Jacques Bros for helpful remarks on analyticity problem in QED. Many thanks to dr Remco Zegers

for helpful correspondance on asymmetries.

\section{References}

[1] J.W.Chen and X.Ji, Phys. Rev. Lett. 86 (2001) 4239.

[2] S.-L.Zhu et al., Phys. Rev. C 64 (2001) 035502. 
[3] P.F.Bedaque and M.J.Savage, Phys. Rev. C 62 (2001) 018501.

[4] J.W.Chen, T.D.Cohen and C.W.Kao, Phys. Rev. C 64 (2001) 055206.

[5] I.B.Khriplovich and R.V.Korkin, Nucl. Phys. A 690 (2001) 610.

[6] J.Ahrens et al., Phys. Rev. Lett. 84 (2000) 5950.

[7] M.Fujiwara et al., Acta Phys. Pol. B 29 (1998) 141.

[8] T.Nakano et al., Nucl. Phys. A 629 (1998) 559c.

[9] M.Fujiwara, Nucl. Phys. News 11 (2001) 28.

[10] O.Bartalini et al. Nucl.Phys. A 699 (2002) 218c.

[11] B.Desplanques, J.F.Donoghue, B.R.Holstein, Ann. Phys. 124 (1980) 449.

[12] S.A.Page et al., Phys. Rev. C 35 (1987) 1119.

[13] M.Bini et al., Phys. Rev. C 38 (1988) 1195.

[14] C.S.Wood et al., Science 275 (1997) 1759.

[15] S.P.Li, E.M.Henley, W-Y.P.Hwang, Ann. Phys. 143 (1982) 372.

[16] R.M.Woloshyn, Can.J.Phys. 57 (1979) 809.

[17] D.J.Almond, Nucl. Phys. B 11 (1969) 277.

[18] K.J.Kim, N.P.H.Dass, Nucl. Phys. B 113 (1976) 336.

[19] K.B.V.Kumar, J.A.McGovern, M.C.Birse, Phys. Lett. B 479 (2000) 167.

[20] X.Ji, C.W.Kao, J.Osborne, Phys. Rev. D 61 (2000) 074003.

[21] G.C.Gellas, T.R.Hemmert, U.G.Meissner, Phys. Rev. Lett. 85 (2000) 14.

G.C.Gellas, T.R.Hemmert, U.G.Meissner, Phys. Rev. Lett. 86 (2001) 3205.

[22] M.C.Birse, X.Ji, J.A.McGovern, Phys. Rev. Lett. 86 (2001) 3204.

[23] O.Hanstein, D.Drechsel, L.Tiator, Nucl. Phys. A 632 (1998) 561.

[24] S.B.Gerasimow, Yad.Fiz. 2 (1965) 598.

[25] S.D.Drell, A.C.Hearn Phys. Rev. Lett. 16 (1966) 908.

[26] S.Ragusa, Phys. Rev. D 47 (1993) 3757.

[27] S.Kondratyuk, O.Scholten, Phys. Rev. C 65 (2002) 038201.

[28] M.J.G.Veltman, Physica 29 (1963) 186.

[29] W.Beenakker, F.A.Berends, A.P.Chapovsky, Nucl. Phys. B 573 (2000) 503. 
[30] D.Buchholz, M.Porrman, U.Stein, Phys. Lett. B 267 (1991) 377.

[31] J.Frohlich, G.Morchio, F.Strocchi, Ann. Phys. 119 (1979) 241.

[32] R.Oehme, Int.J.Mod.Phys. A 10 (1995) 1995.

[33] A.Martin, Scattering Theory: Unitarity, Analyticity and Crossing, Lecture Notes in Physics Vol.3, Springer-Verlag (1969).

[34] C.Itzykson, J.B.Zuber, Quantum Field Theory, ch.5, McGraw-Hill, (1980).

[35] A.Pais, Nuovo Cim. A 53 (1968) 433.

[36] I.B.Khriplovich et al., Sov. Phys. JETP 82 (1996) 616.

[37] I.B. Khriplovich, private communication.

[38] K.Kurek, L.Łukaszuk, unpublished.

[39] M.Gluck, E. Reya, and I.Schienbein, Phys. Rev. D 60 (1999) 054019.

[40] B.Lampe, E.Reya, Phys. Rep. 332 (2000) 1. 